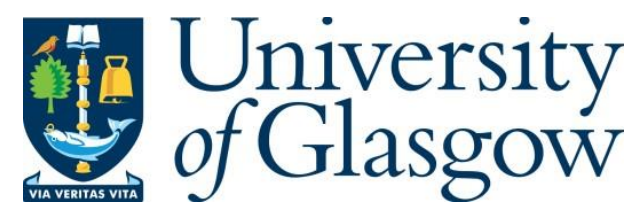

Awad, H., Al-Hamidhi, S., El Hussein, A.-R. M., Yousif, Y. M. z., Taha, K. M., Salih, D. A., Weir, W. and Babiker, H. (2018) Theileria lestoquardi in Sudan is highly diverse and genetically distinct from that in Oman. Infection, Genetics and Evolution, 62, pp. 46-52. (doi:10.1016/j.meegid.2018.04.014)

There may be differences between this version and the published version. You are advised to consult the publisher's version if you wish to cite from it.

http://eprints.gla.ac.uk/161509/

Deposited on: 2 May 2018

Enlighten - Research publications by members of the University of Glasgow http://eprints.gla.ac.uk 


\section{Theileria lestoquardi in Sudan is highly diverse and genetically distinct from that in Oman}

Hoyam Awad ${ }^{1}$, Salama Al-Hamidhi ${ }^{1}$, El Hussein AM $^{2}$, Younis Mohammed zein Yousif ${ }^{3}$, Khalid M Taha ${ }^{2}$, Salih DA ${ }^{2}$, WilliamWeir ${ }^{4}$, Hamza A. Babiker ${ }^{1,5^{*}}$

${ }^{1}$ Department of Biochemistry, College of Medicine and Health Sciences, Sultan Qaboos University, P.O. Box 35, Postal Code 123, Al-Khod, Sultanate of Oman

${ }^{2}$ Animal Resources Research Corporation Khartoum Sudan

${ }^{3}$ Sennar Regional Veterinary Research Laboratory, Sennar, Sudan

${ }^{4}$ School of Veterinary Medicine, College of Medical, Veterinary and Life Sciences, University of Glasgow, UK

${ }^{5}$ Institute of Immunology and Infection Research, School of Biological Sciences, Ashworth Laboratories, University of Edinburgh, Edinburgh, UK

*Corresponding author's Address: Biochemistry Department, Faculty of Medicine, Sultan Qaboos University, Alkhod, PO Box 35, Muscat, Oman. E-mail: H.babiker@ed.ac.uk 


\begin{abstract}
Malignant ovine theileriosis is a severe tick-borne protozoan disease of sheep and other small ruminants which is widespread in sub-Saharan Africa and the Middle East. The disease is of considerable economic importance in Sudan as the export of livestock provides a major contribution to the gross domestic product of this country. Molecular surveys have demonstrated a high prevalence of sub-clinical infections of Theileria lestoquardi, the causative agent, among small ruminants. No information is currently available on the extent of genetic diversity and genetic exchange among parasites in different areas of the country. The present study used a panel of T. lestoquardi specific micro- and mini-satellite genetic markers to assess diversity of parasites in Sudan (Africa) and compared it to that of the parasite population in Oman (Asia). A moderate level of genetic diversity was observed among parasites in Sudan, similar to that previously documented among parasites in Oman. However, a higher level of mixed-genotype infection was identified in Sudanese animals compared to Omani animals, consistent with a higher rate of tick transmission. In addition, the T. lestoquardi genotypes detected in these two countries form genetically distinct groups. The results of this work highlight the need for analysis of T. lestoquardi populations in other endemic areas in the region to inform on novel approaches for controlling malignant theileriosis.
\end{abstract}

Keywords: Theileria lestoquardi, population genetics, Sudan, Oman 


\section{Introduction}

Theileriosis is a tick-borne disease caused by protozoan parasites of the genus Theileria. These parasites infect a wide range of domestic and wild animals and are transmitted by ixodid ticks of several genera including Amblyomma, Haemaphysalis, Hyalomma and Rhipicephalus. The disease is a threat to the agricultural industry of many countries in subSaharan Africa and Asia because of the associated animal mortality, reduction in animal productivity and constraints to livestock improvement (Bishop et al., 2004). Globally, the most economically important species affecting cattle are T. parva and T. annulata, which induce leukocyte transformation and cause East Coast fever and tropical theileriosis, respectively. Theileria lestoquardi is the most pathogenic Theileria species infecting sheep, and to a lesser degree goats, causing malignant ovine theileriosis (Muleya et al., 2012).

Malignant ovine theileriosis (MOT) was first described in Egypt in an imported animal from Sudan (Littlewood, 1915), and then reported in Iraq (Latif et al., 1977), India (Sisodia, 1981), Sudan (El Ghali \& El Hussein, 1995), Turkey (Sayin et al., 1997), Iran (Spitalska et al., 2005), Saudi Arabia (El-Azazy et al., 2001) and the Sultanate of Oman (Tageldin et al., 2005; Shayan et al., 2011). However, it has not been reported in Jordan (Sherkov et al., 1977) or Israel (Pipano, 1991). Although molecular surveys have confirmed the presence of T. lestoquardi in Iran (Ghaemi et al., 2012), Sudan (Salih et al., 2003; El Imam \& Taha, 2015) and the Sultanate of Oman (Tageldin et al., 2005), there is a paucity of information on the distribution of this pathogen. In Oman, local sheep breeds are highly susceptible to ovine theileriosis caused by both T. lestoquardi and T. ovis (Al-Fahdi et al., 2017) with a large proportion of sheep hosting mixed infections with these species along with T. annulata (AlWeheibi, 2011). A recent country-wide molecular survey revealed a high prevalence of T. lestoquardi among sheep $(22 \%)$ and a low prevalence among goats $(0.5 \%)$ (Al-Fahdi et al., 2017).

Theileria spp. that infect livestock are common in central Sudan. The extraordinary climatic and ecological diversity in Sudan, ranging from the desert zone in the north to wooded savannahs in the south together with a wide range of domestic and wildlife hosts, accommodate the biological requirements of a variety of tick species (Elghali \& Hassan, 2012). Amblyomma exornatum, Rhipicephalus sanguineus and nine Hyalomma spp. are common in northern Sudan (Hoogstraal, 1956), with H. anatolicum the most prevalent (A Elghali \& Hassan, 2012). Theileria lestoquardi and T. annulata are known to be widespread across the country (Salih et al., 2003). Serological testing, using the schizont antigen indirect fluorescent antibody test, revealed a high T. lestoquardi infection rate of $16.3 \%$ among sheep in the main grazing areas in Sudan. These findings were later confirmed by surveys in multiple sites in central and northern areas of the country using reverse line blot (RLB) analysis (El Imam \& Taha, 2015). Recent surveys have demonstrated the spread of T. lestoquardi into Western Sudan, in areas where MOT has not been reported previously, with a high infection rate among both goats (41\%) and sheep (69\%) (Osman et al., 2017).

Population genetic analysis of Theileria parasites can provide important information about the epidemiology of disease and may facilitate the development of rational control approaches. Genomic sequence analysis has allowed the identification of putatively neutral, polymorphic micro- and mini-satellite markers for economically important Theileria spp. which has facilitated population genetic analysis of T. parva (Oura et al., 2003) and 
T. annulata (Weir et al., 2007; Al-Hamidhi et al., 2015; Gomes et al., 2016) in a number of endemic regions. More recently, a set of genetic markers has been established for T. lestoquardi and, at present, only one Omani study has applied these markers to examining diversity in field populations of the parasite (Al-Hamidhi et al., 2016). However, a recent study has examined genetic diversity of T. lestoquardi in Sudan using micro- and minisatellites identified in the T. annulata genome (Ali et al., 2017). These two species are phylogenetically closely related and, for this reason, a proportion of $T$. annulata markers can also be used to genotype T. lestoquardi isolates. However, T. annulata markers may not be appropriate for analysis of T. lestoquardi in the field due to the frequent occurrence of mixed species infections in sheep involving T. lestoquardi and T. annulata (Taha et al., 2013; AlFahdi et al., 2017). For this reason, the present study employs a panel of T. lestoquardispecific markers. This approach negates any issues of co-infection with related Theileria species and can allow a credible estimate of the level of diversity within and divergence between populations (Al-Hamidhi et al., 2016).

The present study used T. lestoquardi-specific markers distributed over the four chromosomes for analysis of natural T. lestoquardi populations in Sudan, where MOT is a major problem (El Imam \& Taha, 2015). The data from Sudan was then compared to that from Oman in order to assess the level of genetic differentiation between T. lestoquardi populations in these exemplar African and Asian countries. This provides the first comparative analysis on population structure of T. lestoquardi on sites that are widely geographically separated.

\section{Materials and Methods}

\subsection{Study site and parasite samples}

One hundred and ninety-one blood samples were collected from apparently healthy sheep over a period of one year in $2013(\mathrm{n}=81)$ and in October $2016(\mathrm{n}=110)$ in the River Nile state (Aldamer and Atbra Provinces) in northern Sudan (Figure 1). The samples were collected from adult sheep; the majority of samples collected in 2016 were from ten sheep flocks grazing sheep $(n=80)$ while 30 samples were collected from three farms where the livestock were kept indoors. The River Nile state is characterised by a hot desert climate. The annual average rainfall is $60 \mathrm{~mm}$, which mostly falls between July and August, and the average relative humidity is $29 \%$. Grazing is limited to seasonal watercourses and low-lying areas following sporadic rain showers. Hyalomma anatolicum ticks have been detected in the River Nile state, where $74 \%$ of the ticks feeding on sheep were found to be $H$. anatolicum (Salih et al., 2004; Ahmed et al., 2005).

\subsection{Identification of Theileria species}

DNA was extracted using a Qiagen QIAamp DNA mini Kit (Qiagen, Germany) following the manufacturer's instructions and stored at $-20{ }^{\circ} \mathrm{C}$. Theileria genus-positive samples were identified by polymerase chain reaction (PCR) using pan-Theileria primers, targeting the $18 \mathrm{~S}$ rRNA locus: F 5'-GGCGTTTATTAGACCTAAAACCAAAC-3' and R 5'-TTTGAGCACTCTAATTTTCTCAAAGT-3' (Al-Hamidhi et al., 2016). A total reaction volume of $25 \mu \mathrm{l}$ included $2 \mu \mathrm{l}$ of template DNA, $25 \mathrm{mM} \mathrm{MgCl} 2,10$ pmol of each primer, $1 \mathrm{U}$ DNA Taq polymerase and $10 \mathrm{mM}$ dNTPs. The PCR temperature profile was as follows: denaturation at $95{ }^{\circ} \mathrm{C}$ for 5 minutes, 35 cycles at $95{ }^{\circ} \mathrm{C}$ for 30 seconds, $58{ }^{\circ} \mathrm{C}$ for 30 seconds 
and $65{ }^{\circ} \mathrm{C}$ for 30 seconds, followed by a final extension step at $65{ }^{\circ} \mathrm{C}$ for 5 minutes. The amplified products were then separated on a $2 \%$ ethidium bromide pre-stained agarose gel. To differentiate between the common Theileria species in Oman (Theileria annulata, T. lestoquardi and T. ovis), PCR products were subjected to restriction fragment length polymorphism (RFLP) analysis with HpaII restriction enzyme (Biolab, UK), as described elsewhere (Al-Hamidhi et al., 2015).

\subsection{Micro- and mini-satellite genotyping}

A panel of nine polymorphic micro- and mini-satellite markers designed for the genetic analysis of T. lestoquardi (Al-Hamidhi et al., 2016) was used to genotype each sample. The forward PCR primer in each marker set was labeled with a fluorescent dye (HEX) at the 5' end. PCR was carried out in a total volume of $25 \mu \mathrm{l}$ containing $2 \mu \mathrm{l}$ of template DNA, $1 \mu \mathrm{l}$ of each primer (10 pmol), $1 \mathrm{U}$ of Taq DNA polymerase (Biolab, UK) and $10 \mathrm{mM}$ dNTPs. PCR amplification was carried out at $95^{\circ} \mathrm{C}$ for 5 minutes, then 32 cycles of $95{ }^{\circ} \mathrm{C}$ for 30 seconds, $42-62{ }^{\circ} \mathrm{C}$ for 30 seconds and $65{ }^{\circ} \mathrm{C}$ for 30 seconds followed by a final extension step at $65{ }^{\circ} \mathrm{C}$ for 5 minutes. PCR products were then denatured at $95^{\circ} \mathrm{C}$ for 5 minutes and subjected to capillary electrophoresis on an ABI3130 xl Genetic Analyser (Applied Biosystems, UK). DNA fragment sizes were analysed relative to the ROX-labeled GS500 set of size-standards (Applied Biosystems) using Genemapper software (Applied Biosystems).

Multiple products from a single PCR reaction indicated the presence of a mixture of genotypes. To determine the relative concentration of each allele/amplicon, the area under each peak was measured. In this way, the predominant allele at each locus was identified for each sample and this data was combined to generate a multi-locus genotype (MLG) that represents an estimate of the most abundant genotype in each sample. The MLG data was then used to determine a number of indices including genetic diversity, linkage disequilibrium and population differentiation.

\subsection{Genetic analysis}

The MLG dataset was compared to a previously generated dataset from $T$. lestoquardi isolates in Oman (Al-Hamidhi et al., 2016) in order to determine the extent of diversity and genetic relatedness among the two populations. Tandem software (Matschiner \& Salzburger, 2009) was utilised to facilitate consistent allele-calling and, since most loci were located in exons, a 3 bp repeat unit was used to differentiate alleles. Genetic diversity parameters were calculated for the entire dataset using GenAlex v6.5 (Peakall \& Smouse, 2012). This included determining the number of alleles per locus and expected heterozygosity $\left(H_{e}\right)$. These two parameters were used to assess the level of polymorphism at each locus and determine diversity overall and within the sub-populations. Expected heterozygosity was calculated using the formula for 'unbiased heterozygosity' also termed haploid genetic diversity, $H_{e}=[\mathrm{n} /(\mathrm{n}-1)]\left[1-\sum \mathrm{p}^{2}\right]$, where $n$ is the number of isolates and $p$ is the frequency of each different allele at a locus (Anon, 1996).

To determine whether T. lestoquardi parasites in Aldamer and Atbra Provinces comprised a single panmictic population with a high degree of genetic exchange, multi-locus linkage disequilibrium (LD) of the alleles at pairs of loci was measured using the standard index of 
association $\left(\mathrm{I}_{\mathrm{A}}^{\mathrm{S}}\right)$. Both $\mathrm{I}_{\mathrm{A}}^{\mathrm{S}}$ and variance of data were calculated using the program LIAN version 3.5 (Haubold \& Hudson, 2000).

Population differentiation was assessed by estimating Wright's $F_{S T}$ index using the Fstat computer package Version 2.9.3.2. Two estimators of $F_{S T}\left(G^{\prime}\right.$ ST and $\theta$ ) (Cockerham \& Weir, 1984; Nei, 1987) were used to estimate genetic differentiation between sub-populations. Principal Co-ordinate analysis (PCoA) was performed using GeneAlex6 in order to visualise the relationships between MLGs. In addition, the partitioning of genetic diversity among and between populations was investigated using analysis of molecular variance (AMOVA) (Excoffier et al., 1992).

The software Structure V. 2.3.4 was used to deduce the underlying structure of parasite population and determine how many sub-populations it comprised. This method detects clusters without prior information on the origin of samples. Ten iterations for $\mathrm{K}=2$ to $\mathrm{K}=5$ ( $\mathrm{K}$ being the number of clusters) were run, each with a burn-in period of 10,000 steps and then 20,000 Markov Chain Monte Carlo (MCMC) iterations. In addition, the optimal number of clusters was assessed using the program Structurama (Huelsenbeck et al., 2011).

\subsection{Multiplicity of infection}

Multiplicity of infection (MOI), defined as the presence of multiple genotypes in an individual, was determined by the presence of more than one allele at a locus in a sample. To avoid over-estimation of low abundance alleles, only minor alleles having a peak height $>33 \%$ of the corresponding predominant allele were accepted. This eliminated spurious peaks in the vicinity of major peaks, which if unaccounted for could overestimate the presence of rare alleles thus artificially inflating the calculated MOI. The mean number of alleles across the nine loci in each sample was calculated and this index value was used to represent the MOI within each sample. The overall mean for the index values for each sample was then calculated.

\section{Results}

\subsection{Diversity of micro- and mini-satellites}

A total of 191 apparently healthy sheep in Aldamer and Atbra Provinces, Sudan, were screened for the presence of T. lestoquardi by PCR-RFLP of the $18 S$ rRNA locus. Theileria lestoquardi was detected in a total of 98 animals (51\%). For samples collected in 2016, a total of 85 of $110(77.2 \%)$ were found to be positive for T. lestoquardi. This comprised 12 positives among 30 animals kept indoors (40\%) and 73 of 80 grazing animals $(91.2 \%)$. In 2013, 13 of 81 animals (16\%) were found to be positive for T. lestoquardi. Of the 98 T. lestoquardi isolates, 90 (13 from 2013 and 77 from 2016) were successfully genotyped using nine species-specific micro- and mini-satellites (supplementary Table 1).

A high level of polymorphism was observed among the ninety T. lestoquardi isolates. The total number of alleles in the population sample ranged from 2 for TL_MS25 to 17 for TL_MS13 (Table 1). The number of alleles per locus within a single sample ranged from one to seven (Table 1). Seven alleles were detected in a single sample using the marker TL_MS13, which was the most polymorphic of the loci analysed, possessing 17 defined 
alleles across the entire dataset. A high-level of polymorphism was observed among six other loci (TL_MS281, TL_MS280, TL_MS04, TL_MS16, TL_MS07 and TL_MS18) (Figure 2) and a lower level of allelic polymorphism was observed for TL_MS 19 and TL_MS25.

The average heterozygosity among T. lestoquardi parasites in the Aldamer and Atbra provinces in Sudan was found to be moderate $\left(H_{e}=0.572\right)$, ranging from 0.355 to 0.816 . This is comparable to that reported for the parasite population in Oman $\left(H_{e}=0.582\right)$, examined using the same panel of markers (Al-Hamidhi et al., 2016).

\subsection{Multiplicity of infection}

Eighty-three $(92 \%)$ of the 90 Sudanese T. lestoquardi isolates analysed carried multiple parasite genotypes. The minimum number of alleles per locus per isolate ranged between 1 and 7 alleles, with average of $3(\mathrm{SD} \pm 1.3)$. In contrast, among Omani isolates, the number of alleles per locus ranged between 1 and 6, with an average of 2.6 (SD \pm 0.9 ). The overall prevalence of isolates showing a MOI was higher in Sudan (92\%) than in Oman (44\%) $(\mathrm{P}=0.0001)$.

\subsection{Linkage disequilibrium}

LD was estimated using the standard index of association $\left(\mathrm{I}_{\mathrm{A}}^{\mathrm{S}}\right)$ to investigate whether the high diversity observed in T. lestoquardi in Sudan could be explained by a high rate of recombination, a consequence of sexual reproduction. Linkage analysis was first performed by combining data from samples collected in 2013 and 2016. In addition, linkage analysis was performed for the combined population in Sudan and Oman. When all samples from Sudan were treated as a single population, an $\mathrm{I}^{\mathrm{S}}{ }_{\mathrm{A}}$ value of 0.116 and a $\mathrm{V}_{\mathrm{D}}$ value (3.812) greater than L (2.171) was obtained, indicating LD (Table 2). Similar results were obtained when each year in Sudan was examined separately (Table 2). The combined Sudanese and Omani dataset showed a slightly higher degree of association between loci than for the Sudan dataset alone $\left(\mathrm{I}_{\mathrm{A}}^{\mathrm{S}}=0.124\right)$.

\subsection{Population sub-structuring}

To measure the level of genetic differentiation within T. lestoquardi in Sudan and between Sudanese and Omani populations, FST values were estimated. Two estimates of F $_{\text {ST }}\left(\mathrm{G}_{\text {ST }}^{\prime}\right.$ and 0) (Cockerham \& Weir, 1984; Nei, 1987) were calculated. No differentiation was observed between the T. lestoquardi population samples collected in Sudan in 2013 and 2016 $\left(\mathrm{G}^{\prime} \mathrm{ST}=0.029\right.$ and $\left.\theta=0.027\right)$. Additionally, no genetic differentiation was detected between T. lestoquardi populations in grazing sheep and those kept indoors $(\mathrm{FST}=0.011)$. However, a high level of differentiation $\left(\mathrm{G}^{\prime}{ }_{\mathrm{ST}}=0.295\right.$ and $\left.\theta=0.295\right)$ was evident between parasite populations in Oman and Sudan.

The above finding was supported by subsequent PCoA analysis. There was no evidence of differential clustering of parasite samples collected in 2013 and 2016 (Figure 3A). However, discrete clustering of parasites from Sudan and Oman was evident, indicating geographical isolation may be associated with genetic isolation (Figure 3B). The amount of molecular variation obtained represented by the first and second axes was $45.03 \%$ and $12.95 \%$, respectively (Figure 3B), indicating that this diagram is able to illustrate much of the underlying genetic variation in the dataset. Analysis of molecular variance (AMOVA), 
suggested that most of the genetic variation, $72 \%$, was contained within sub-populations with only $28 \%$ explained by differences between sub-populations.

Structure analysis clearly indicated the presence of two discrete clusters representing underlying sub-populations $(K=2)$. Sudanese MLGs formed one cluster while Omani MLGs formed another (Figure 4).

\section{Discussion.}

The present study examined the diversity of T. lestoquardi using species-specific mini- and micro-satellites and assessed the population structure of the parasites in Sudan, comparing it to that in Oman. This is the first comparative genetic analysis of T. lestoquardi parasites in geographically separated sites. The parasites in Sudan and Oman were shown to form genetically distinct groups. However, the extent of diversity among T. lestoquardi in Sudan $\left(H_{e}=0.572\right)$ was similar to that documented among parasites in Oman $\left(H_{e}=0.582\right)$. Nonetheless, a higher prevalence of MOI was inferred among isolates from Sudan (92\%) compared to those from Oman (44\%).

The presence of a high level of genotypic diversity and a high prevalence of MOI among infected sheep in Aldamer and Atbra provinces, northern Sudan, is indicative of intense transmission. Previous surveys demonstrated high rates of tick infestation in this area, as well as other sites in the country (Osman et al., 2017). A higher rate of tick infestation and transmission may be linked to a significantly higher MOI in Sudan compared to that in Oman. MOI and mean number of genotypes within individual hosts reflect the transmission intensity, abundance of vector (ticks) and the level of host (sheep) infestation (Weir et al., 2011). Hyalomma anatolicum is the most abundant vector of Theileria spp. both in Sudan (El Hussein et al., 2012) and Oman (Al-Fahdi et al., 2017). A previous study in Sudan reported a high rate of Theileria infection in H. anatolicum, ranging from $8.6 \%$ to $49.6 \%$ (Salih et al., 2004; Osman et al., 2017), however similar information is not available for Oman. Such a high vector infection rate can result in a high level of transmission and super-infection that can lead to the acquisition of multiple genotypes among vertebrate hosts (Conway et al., 1992). This may explain why MOI was two-fold higher in Sudan than that in Oman, with almost every infected animal harbouring more than one parasite genotype. The high rate of asymptomatic carriage of $T$. lestoquardi among local sheep coupled with the elevated rate of transmission presumably, allow continuous inoculation of new genotypes to local animals and high prevalence of disease. However, it is of some interest that each animal contained only a limited number of genotypes, at least on the days of sampling, with an average of 3 genotypes/infection in Sudan compared to 2.6 genotypes/infection in Oman. This may be attributed to the fact that some host factors could limit acquisition of multiple clones in the vertebrate hosts of apicomplexan parasites, such as age, due to mounting immunity resulting from increased exposure to infection (Weir et al., 2011; Pacheco et al., 2016) and breed/ethnic group (Pacheco et al., 2016). Acquired immunity and prevalence of MOI are age structured in malaria endemic areas, suggesting that acquired immunity may influence the risk of subsequent infection (Smith et al., 1999). Additional factors that can limit MOI in infected sheep may include within-host competitive interaction between multiple genotypes (Huijben et al., 2011) or presence of some genotypes below the PCR detection threshold. Similar observations of limited MOI in a single infection have been reported for the malaria parasite 
P. falciparum in countries with high malaria transmission (Ntoumi et al., 1995; Smith et al., 1999). However, MOI has been found to be a reliable indicator for success of control efforts, and a significant reduction can reflect a reduction in the rate of transmission and superinfection locally (Nkhoma et al., 2013). Therefore, control efforts that cut transmission can reduce rates of MOI in Theileria endemic areas and the likelihood of emergence of novel recombinant genotypes that can spread the disease to less immune competent animals.

The moderate diversity among T. lestoquardi reported in the present study in Sudan, and that previously reported in Oman (Al-Hamidhi et al., 2016), was lower than that observed in T. annulata in different endemic sites, such as Portugal (Gomes et al., 2016), Turkey and Tunisia (Weir et al., 2011). However, it is important to note that the set of markers used to define T. annulata genotypes is different from those used in T. lestoquardi and so the results at each locus are not directly comparable. Nevertheless, given that each set of markers was selected from their respective genome using very similar criteria, they may be taken as representative sub-sets of highly polymorphic markers from each species. Compared to T. annulata in Tunisia and Turkey, lower genetic diversity was found in T. lestoquardi in both Sudan $(\mathrm{P}<0.01)$ and Oman $(\mathrm{P}<0.001)$ (Student's T test). Even in the same region where the two species co-exist, $T$. annulata showed higher diversity than T. lestoquardi (Al-Hamidhi et al., 2016). For example, in two areas of Oman where T. lestoquardi isolates were collected from small ruminants and T. annulata was collected from cattle living in the same farms, lower diversity was noted in T. lestoquardi ( $\mathrm{P}<0.01$ (Batinah) and $\mathrm{P}<0.05$ (Sharqia), Student's T test) (Al-Hamidhi et al., 2016). The generally lower diversity of T. lestoquardi compared to that seen in T. annulata, supports the hypothesis that $T$. lestoquardi has evolved from an ancestral T. annulata, and that parasite speciation occurred as the parasite adapted to the small ruminant host (Schnittger et al., 2000). This hypothesis is consistent with the high identity at the $18 \mathrm{~S}$ rRNA locus between T. lestoquardi and T. annulata (Schnittger et al., 2003). The feature that stands out in the present study in both T. lestoquardi populations in Africa and Asia is the lower genetic diversity compared to T. annulata (Al-Hamidhi et al., 2016). Comparative genomic analysis, using genome sequence data of T. lestoquardi, will shed more light on this hypothesis (Professor Arnab Pain, King Abdullah University of Science \& Technology, Thuwal, Kingdom of Saudi Arabia; Personal communication;).

Despite the high rate of infection and MOI observed among T. lestoquardi isolates in Sudan, a significant association between genetically unlinked loci was observed, implying LD. This LD is not explained by sub-structuring between parasites isolated in 2013 and 2016, as both PCoA and FST analysis showed no evidence of between-year population variation. It is possible that factors such as influx of new genotypes (Wahlund effect) or an epidemic population structure may cause the observed LD. A lower level of LD has been observed within T. lestoquardi in some regions in Oman, even at the level of a single farm (Al-Hamidhi et al., 2016). Similar findings have been observed among T. annulata in Tunisia, Turkey (Weir et al., 2011) and Portugal (Gomes et al., 2016). Recombination, as measured by genetic linkage, in the Portuguese T. annulata population is undoubtedly frequent and is at a broadly similar level to that occurring in Tunisia. Although animals are kept exclusively on farms and are not free-roaming, it is known that there are movements of cattle from farm to farm particularly in neighboring regions (Baptista \& Nunes, 2007). Our data suggests that sheep movement is insufficient to homogenise the present parasite population and that geographical 
sub-structuring is maintained at the country level. The parasite population is essentially panmictic with only a minor disturbance showed by the low levels of $\mathrm{I}_{\mathrm{A}}^{\mathrm{S}}$ and only a limited level of LD.

Very low levels of genetic differentiation were detected between T. lestoquardi parasites collected in 2013 and 2016 in Sudan, with a pair-wise FST value of 0.029. However, a high level of genetic differentiation was measured between T. lestoquardi parasites in Sudan and Oman, with a FST value of 0.295. This is consistent with similar findings of genetic differentiation in populations of other Theileria species such as T. annulata (Gomes et al., 2016) and T. parva (Muwanika et al., 2016), separated by geographical barriers. A higher level of differentiation has been documented between T. annulata in Oman and Turkey $\left(\mathrm{F}_{\mathrm{ST}}=0.102\right)$ than Oman and Tunisia $\left(\mathrm{F}_{\mathrm{ST}}=0.063\right)(\mathrm{Al}-\mathrm{Hamidhi}$ et al., 2015). One hypothesis to explain this finding would be more frequent demographic exchange between livestock populations in Oman and Tunisia than Oman and Turkey (Al-Hamidhi et al., 2015). The differentiation between populations in widely separated countries is consistent with geographical and trade barriers hindering gene flow. Nonetheless, there was some overlap between parasites in Sudan and Oman, and complete separation between the parasite populations in the two countries was not observed (Figure 3). Similarly, incomplete separation has been observed in T. annulata from widely separated countries (Weir et al., 2007; Al-Hamidhi et al., 2015). This suggests that despite ecological and epidemiological barriers between the parasite populations, there are likely to have been some gene flow between them, and this may be related to movement of animals since tick migration can be ruled out in absence of host movement. Alternatively, the two populations might have not been separated for enough evolutionary time for them to drift apart.

In summary, a moderate level of genetic diversity was observed in the T. lestoquardi population in sheep in the Aldamer and Atbra provinces in Sudan. There was a high level of MOI in infected animals and this indicates a large parasite reservoir and gene pool. The lower extent of MOI observed among T. lestoquardi in Oman compared to Sudan can be attributed to differences in epidemiology in each country which may be related to the abundance and dynamics of the tick vector.

\section{Acknowledgements}

We are grateful to the farmers and the staff of the Ministry of Agriculture in Sudan and the staff of the Ministry of Agriculture and Fisheries, Oman, for their support with field surveys. We appreciate the support of the technical staff of the Biochemistry Department, Sultan Qaboos University, Oman.

\section{Financial support}

This study was supported by project GCC Co-Funding Research Grant CL/SQU/GCC/17/01 and a student scholarship from HA, Sultan Qaboos University, Oman. 
Figure 1. Theileria lestoquardi sampling sites in River Nile state, Sudan

Figure 2 (A \& B). The frequency of alleles, as a percentage (\%), is shown for two microsatellites, TL_MS07 and TL_MS13, in Sudanese and Omani parasite populations. The size of each allele (in $\mathrm{bp}$ ) is given on the $\mathrm{x}$ - axis.

Figure 3 (A \& B): Principal Co-ordinate analysis (PCoA) of T. lestoquardi genotypes from Sudan and Oman. The amount of variation in the dataset represented by each axis is shown as a percentage.

Figure 4: 'Structure' analysis of T. lestoquardi genotypes from Sudan and Oman. The presence of two sub-populations was inferred $(K=2)$. 


\section{Reference:}

Ahmed, B. M., El-Hussein, A. M., \& El-Khider, A. O. (2005). Some observations on ticks (Acari: Ixodidae) infesting sheep in River Nile Province of Northern Sudan. The Onderstepoort journal of veterinary research, 72(3), 239-243 .

Al-Fahdi, A., Alqamashoui, B., Al-Hamidhi, S., Kose, O., Tageldin, M. H., Bobade, P., . . . Babiker, H. (2017). Molecular surveillance of Theileria parasites of livestock in Oman. Ticks and Tick-borne Diseases, 8(5), 741-748. doi: https://doi.org/10.1016/j.ttbdis.2017.05.008

Al-Hamidhi, S., H Tageldin, M., Weir, W., Al-Fahdi, A., Johnson, E. H., Bobade, P., . . . Babiker, H. (2015). Genetic Diversity and Population Structure of Theileria annulata in Oman. PloS one, 10(10), e0139581. doi:10.1371/journal.pone.0139581

Al-Hamidhi, S., Weir, W., Kinnaird, J., Tageledin, M., Beja-Pereira, A., Morrison, I., . . . Babiker, H. A. (2016). Theileria lestoquardi displays reduced genetic diversity relative to sympatric Theileria annulata in Oman. Infection, Genetics and Evolution, 43, 297-306. doi: http://dx.doi.org/10.1016/j.meegid.2016.05.007

Al-Weheibi, H. (2011). Studies on Theileriosis in Indigenous Sheep in Al-Batinah and A'Dakheliya Regions of Oman. Sultan Qaboos University .

Ali, A. M., Salih, D. A., Njahira, M. N., Hassan, S. K., El Hussein, A. M., Liu, Z., . . Skilton, R. A. (2017). Genotyping of Theileria lestoquardi from sheep and goats in Sudan to support control of Malignant Ovine Theileriosis. Veterinary Parasitology, 239, 7-14. doi: https://doi.org/10.1016/j.vetpar.2017.04.005

Anon, A. (1996). The Evaluation of Forensic DNA Evidence. Proceedings of the National Academy of Sciences, 94(11), 5498-5 .500

Babiker, H. A., \& Walliker, D. (1997). Current views on the population structure of plasmodium falciparum: Implications for control. Parasitology Today, 13(7), 262-267. doi: http://dx.doi.org/10.1016/S0169-4758(97)01075-2

Baptista, F. M., \& Nunes, T .(2007) .Spatial analysis of cattle movement patterns in Portugal. Veterinaria italiana, 43(3), 611-619.

Bishop, R., A Musoke, S Morzaria, M Gardner, \& Nene, V. (2004). Theileria: Intracellular Protozoan Parasites of Wild and Domestic Ruminants Transmitted by Ixodid Ticks. Parasitology Research, 129(Suppl: S271-83. doi:10.1017/S0031182003004748.) .

Cockerham, C. C., \& Weir, B. S. (1984). Covariances of Relatives Stemming from a Population Undergoing Mixed Self and Random Mating. Biometrics, 40(1), 157-1 .64 doi: $10.2307 / 2530754$

Conway, D. J., Greenwood, B. M., \& McBride, J. S. (1991). The epidemiology of multipleclone Plasmodium falciparum infections in Gambian patients. Parasitology, 103(01), 1-5. doi: doi:10.1017/S0031182000059217

Conway, D. J., Greenwood, B. M., \& McBride, J. S. (1992). Longitudinal study of Plasmodium falciparum polymorphic antigens in a malaria-endemic population. Infection and Immunity, 60(3), 1122-1127 .

El-Azazy, O., El-Metenawy, T., \& Wassef, H. (2001). Hyalomma impeltatum (Acari: Ixodidae) as a potential vector of malignant theileriosis in sheep in Saudi Arabia. Veterinary Parasitology, 99(4), 305-309 . 
El Ghali, A., \& El Hussein, A. (1995). Diseases of livestock in EdDamer Province, River Nile State, Sudan: a two-years retrospective study. Sud J Vet Sci Anim Husb, 34, 37-45 .

El Hussein, A. M., Hassan, S. M., \& Salih, D. A. (2012). Current situation of tropical theileriosis in the Sudan. Parasitology Research, 111(2), 503-508. doi: $10.1007 / \mathrm{s} 00436-012-2951-5$

El Imam, A. H \& ,.Taha, K. M. (2015). Malignant Ovine Theileriosis (Theileria lestoquardi): A Review. Jordan Journal of Biological Sciences Review, 8(3), 165 - 174 .

Elghali, A. A., \& Hassan, S. M. (2012). Ticks infesting animals in the Sudan and Southern Sudan: past and current status. The Onderstepoort journal of veterinary research, 79(1), E1-6.

Excoffier, L., Smouse, P. E., \& Quattro, J. M. (1992). Analysis of molecular variance inferred from metric distances among DNA haplotypes: application to human mitochondrial DNA restriction data. Genetics, 131(2), 479-491 .

Ghaemi, P., Hoghooghi-Rad, N., Shayan, P., \& Eckert, B. (2012). Detection of Theileria orientalis in Iran by semi-nested PCR. Parasitology Research, 110(2), 527-531 .

Gomes, J., Salgueiro, P., Inácio, J., Amaro, A., Pinto, J., Tait, A., . . Weir, W. (2016). Population diversity of Theileria annulata in Portugal. Infection, Genetics and Evolution, 42 .19-14 ,doi: http://dx.doi.org/10.1016/j.meegid.2016.04.023

Haubold, B., \& Hudson, R. R. (2000). LIAN 3.0: detecting linkage disequilibrium in multilocus data. Bioinformatics (Oxford, England), 16(9), 847-849. doi: 10.1093/bioinformatics/16.9.847

Hoogstraal, H. (1956). Notes on African Haemaphysalis ticks. III. The hyrax parasites, H. bequaerti sp. nov., H. orientalis N. and W., 1915 (new combination), and H. cooleyi Bedford, 1929 (Ixodoidea, Ixodidae). The Journal of parasitology, 42(2), 156-172 .

Huelsenbeck, J. P., Andolfatto, P., \& Huelsenbeck, E. T. (2011). Structurama: Bayesian Inference of Population Structure. Evolutionary Bioinformatics Online, 7, 55-59. doi: 10.4137/EBO.S6761

Latif, B., Hewa, N., \& Bakir, F. (1977). Incidence of malignant Theileriosis (Theileria hirci) of sheep in Iraq. Iraqi Journal of Veterinary Medicine .

Littlewood, W. (1915). Annual Report for 1914. Ministry of Agriculture, Egypt, Veterinary Service .

Matschiner, M., \& Salzburger, W. (2009). TANDEM: integrating automated allele binning into genetics and genomics workflows. Bioinformatics (Oxford, England), 25(15), 1982-1983. doi: 10.1093/bioinformatics/btp303

Muleya, W., Namangala, B., Simuunza, M., Nakao, R., Inoue, N., Kimura, T., . . Sawa, H. (2012). Population genetic analysis and sub-structuring of Theileria parva in the northern and eastern parts of Zambia. Parasites \& Vectors, 5, 255-255. doi: 10.1186/1756-3305-5-255

Muwanika, V., Kabi, F., \& Masembe, C. (2016). Population genetic structure of Theileria parva field isolates from indigenous cattle populations of Uganda. Ticks and Tickborne Diseases, 7(2), 291-297. doi: https://doi.org/10.1016/j.ttbdis.2015.11.004

Nei, M. (1987). Molecular Evolutionary Genetics. Columbia University Press, New York .

Nkhoma, S. C., Nair, S., Al-Saai, S., Ashley, E., McGready, R., Phyo, A. P., . . Anderson, T. J. C. (2013). Population genetic correlates of declining transmission in a human pathogen. Molecular Ecology, 22(2), 273-285. doi: 10.1111/mec.12099 
Ntoumi, F., Contamin, H., Rogier, C., Bonnefoy, S., Trape, J.-F., \& Mercereau-Puijalon, O. (1995). Age-dependent carriage of multiple Plasmodium falciparum merozoite surface antigen-2 alleles in asymptomatic malaria infections. The American journal of tropical medicine and hygiene, 52(1), 81-88 .

Osman, T., Ali, A., Hussein, M., El Ghali, A., \& Salih, D. (2017). Investigation on Theileria lestoquardi infection among sheep and goats in Nyala, South Darfur State, Sudan. Insights Vet Sci, 1, 017-023 .

Oura, C .A. L., Odongo, D. O., Lubega, G. W., Spooner, P. R., Tait, A., \& Bishop, R. P. (2003). A panel of microsatellite and minisatellite markers for the characterisation of field isolates of Theileria parva. International Journal for Parasitology, 33(14), 1641.1653doi: http://dx.doi.org/10.1016/S0020-7519(03)00280-7

Pacheco, M. A., Lopez-Perez, M., Vallejo, A. F., Herrera, S., Arévalo-Herrera, M., \& Escalante, A. A. (2016). Multiplicity of Infection and Disease Severity in Plasmodium vivax. PLOS Neglected Tropical Diseases, 10(1), e0004355. doi: 10.1371/journal.pntd.0004355

Peakall, R., \& Smouse, P. E. (2012). GenAlEx 6.5: genetic analysis in Excel. Population genetic software for teaching and research-an update. Bioinformatics (Oxford, England), 28(19), 253 .2539-7doi: 10.1093/bioinformatics/bts460

Pipano, E. (1991). Observations on the seasonal distribution of blood parasites in sheep in Israel. Israel Journal of Veterinary Medicine, 46(1), 37-38 .

Salih, D. A., ElHussein, A. M., Hayat, M., \& Taha, K. M .(2003) .Survey of Theileria lestoquardi antibodies among Sudanese sheep. Veterinary Parasitology, 111(4), 361367. doi: 10.1016/s0304-4017(02)00389-8

Salih, D. A., Hassan, S. M., El Hussein, A. M., \& Jongejan, F. (2004). Preliminary survey of ticks (Acari :Ixodidae) on cattle in northern Sudan. The Onderstepoort journal of veterinary research, 71(4), 319-326.

Sayin, F., Dyncer, S., Karaer, Z., Cakmak, A., Yukary, B., Eren, H., . . Nalbantoglu, S. (1997). Status of the tick-borne diseases in sheep and goats in Turkey. Parassitologia, 39(2), 153-156 .

Schnittger, L., Hong, Y., Jianxun, L., Ludwig, W., Shayan, P., Rahbari, S., . . Ahmed, J. S. (2000). Phylogenetic analysis by rRNA comparison of the highly pathogenic sheepinfecting parasites Theileria lestoquardi and a Theileria species identified in China. Annals of the New York Academy of Sciences, 916, 271-275.

Schnittger, L., Yin, H., Gubbels, M. J., Beyer, D., Niemann, S., Jongejan, F., \& Ahmed, J. S. (2003). Phylogeny of sheep and goat Theileria and Babesia parasites. Parasitology Research, 91(5), 398-406. doi: 10.1007/s00436-003-0979-2

Shayan, P., Ebrahimzadeh, E., Tageldin, M., Amininia, N., \& Eckert, B. (2011). Molecular study of sheep malignant theileriosis at barka region in the sultanate of oman. Iranian journal of parasitology, 6(1), 66-72 .

Sherkov, S., El-Rabei, Y., \& Kakash, L. (1977). A survey of parasitic blood diseases;"'TickBorne Fever" in domestic animals in Jordan. Egypt J Vet Sci, 13, 29-35 .

Sisodia, R. (1981). Present status of sheep theileriasis in India--a review. Livestock adviser .

Smith, T., Felger, I., Beck, H., \& Tanner, M. (1999). Consequences of multiple infection with Plasmodium falciparum in an area of high endemicity. Parassitologia, 41(1-3), 247250 . 
Spitalska, E ,.Namavari, M. M., Hosseini, M. H., Shad-del, F., Amrabadi, O. R., \& Sparagano, O. A. (2005). Molecular surveillance of tick-borne diseases in Iranian small ruminants. Small Ruminant Research, 57(2), 245-248 .

Tageldin, M. H., Fadiya, A. A.-K., Sabra, A. A-.Y., \& Ismaily, S. I. A.-I. (2005). Theileriosis in sheep and goats in the Sultanate of Oman. Tropical Animal Health and Production, 37(6), 491-493. doi: 10.1007/s11250-005-2475-4

Taha, K. M., Salih, D. A., Ali, A. M., Omer, R. A., \& El Hussein, A. M. (2013) .(Naturally occurring infections of cattle with Theileria lestoquardi and sheep with Theileria annulata in the Sudan. Veterinary Parasitology, 191(1-2), 143-145. doi: http://dx.doi.org/10.1016/j.vetpar.2012.08.003

Weir, W., Ben-Miled, L., Karagenç, T ,.Katzer, F., Darghouth, M., Shiels, B., \& Tait, A. (2007). Genetic exchange and sub-structuring in Theileria annulata populations. Molecular and Biochemical Parasitology, 154(2), 170-180. doi: http://dx.doi.org/10.1016/j.molbiopara.2007.04.015

Weir, W ,.Karagenç, T., Gharbi, M., Simuunza, M., Aypak, S., Aysul, N., . . Tait, A. (2011). Population diversity and multiplicity of infection in Theileria annulata. International Journal for Parasitology, 41(2), 193-203. doi: 10.1016/j.ijpara.2010.08.004 